\title{
28. SILICEOUS SPONGE SPICULES FROM DEEP SEA DRILLING PROJECT LEG 931
}

\author{
Kevin McCartney, Florida State University²
}

\begin{abstract}
Abundant siliceous sponge spicules were found in the lower and middle Eocene sediments of Site 605 and the upper Miocene sediments of Site 604 . The assemblages consist mostly of monaxons. Relative abundances of the various types are tabulated. All those from the Miocene sample, and the vast majority of those from the Eocene sample, belong to the Class Demospongea. A small minority of the Eocene spicules are from the Class Hexactinellida.
\end{abstract}

\section{INTRODUCTION}

A diverse assemblage of siliceous sponge spicules was found at DSDP Sites 604 and 605 of Leg 93 off the Atlantic coast of the United States while parts of these sections were being examined for silicoflagellates (McCartney and Wise, this volume) (Fig. 1). Siliceous sponge spicules are sometimes quite abundant in DSDP samples but are seldom discussed; among papers that do so are Dumitrică (1973), Bukry $(1978,1979,1980 a, b)$, and Ivanik (1983). In this study, the spicules are particularly abundant and diverse in an early and middle Eocene interval of Site 605 , where they are one of the most important components of the siliceous sediment. Sponge spicules are less abundant and diverse in a late Miocene interval from Site 604 . At both sites the assemblages are dominated by monaxons, with other types representing only a minor fraction of the total abundance. The vast majority of the spicules are from the Class Demospongea, including all from Site 604 . Only the amphidiscs and hexactines (and the possible hexasters) from Site 605 belong to the Class Hexactinellida.

\section{METHODS}

Raw samples were placed in $100 \mathrm{ml}$ beakers and a small amount of $30 \%$ hydrogen peroxide added; more was added if it strongly effervesced. After 2-4 hr. (or longer if effervescence continued when more hydrogen peroxide was added), the beaker was placed in an ultrasonic cleaner and distilled water was added to about the $25 \mathrm{ml}$ level. $\mathrm{HCl}$ was added and the beaker heated for $30 \mathrm{~min}$. Samples were then centrifuged and decanted twice and washed once more before making strewn slides.

Relative abundance was determined by examining an entire $22 \times$ $40 \mathrm{~mm}$ slide and is reported according to the following scale (Tables 1 and 2): Dominant (D), $>50 \%$ of all spicules; Abundant (A), 10-50\%; Common (C), 1-10\%; Uncommon (U), $0.1-1 \%$ (2-5 per slide); Rare (R), $<0.1 \%$ (1 per slide).

\section{TERMINOLOGY}

Although Ehrenberg (1854) erected a Linnean taxonomy for fossil sponges, a descriptive terminology for isolated spicules is generally used (see Hyman, 1940; Sol-

\footnotetext{
${ }^{1}$ van Hinte, J. E., Wise, S. W., Jr., et al., Init. Repts. DSDP, 93: Washington (U.S. Govt, Printing Office).

2 Address: Department of Geology, Florida State University, Tallahassee, FL 32306-3026.
}

las, 1906; and Bukry, 1978). A usable formal spicule taxonomy is not practical because a single living species may contain a variety of spicule types, and similar spicules can be produced by different species of sponge. Ivanik (1983), however, demonstrates that sponge spicules do have some biostratigraphic and paleoecologic use. The descriptive terms used in this study to tabulate the relative abundances are listed here, with comments where applicable.

\section{Class DEMOSPONGEA Sollas}

\section{Monaxons}

\section{Diactinal (monaxons with similar ends)}

Oxeas (pointed ends; Plate 2, Figs. 1, 9): Very abundant, generally making up $30-40 \%$ of the sponge spicules at Site 605, and 60-70\% at Site 604. Length and relative thickness vary widely and there are no distinguishable patterns of the types found by Ivanik (1983). Specimens can be longer than $500 \mu \mathrm{m}$. Relatively thick, short oxeas are tabulated separately (Plate 2, Fig. 9).

Strongyles (monaxons with rounded ends; Plate 1, Figs. 5-7): Nonspinose strongyles were sporadic at both sites, with a large variation in size and thickness.

Acanthoxeas (spinose oxeas; Plate 2, Fig. 2): Sporadic throughout Site 605, relatively more common at Site 604 . Lengths vary, but the density of nodes on the spicule is generally rather low.

Acanthostrongyles (spinose strongyles; Plate 2, Fig. 4).

\section{Monactinal (monaxons rounded at one end and pointed at the other)}

Styles (no change in thickness at rounded end; Plate 2, Fig. 5, Plate 1, Figs. 2, 3): Common at both sites. Range of variation in size, thickness, and curvature was similar to the oxeas.

Tylostyles (one end pointed, the other knobbed; Plate 2, Figs. 3, 6-7).

Acanthostyles (spinose styles; Plate 1, Figs. 3, 4).

Acanthotylostyles (spinose tylostyles; Plate 2, Fig. 8): some forms had spines only at the knobbed end.

"Serrated-spines" (Plate 1, Fig. 1): Associated with the sponge spicules throughout Site 605 was a serrated form originally thought to be poriferan, but since deter- 


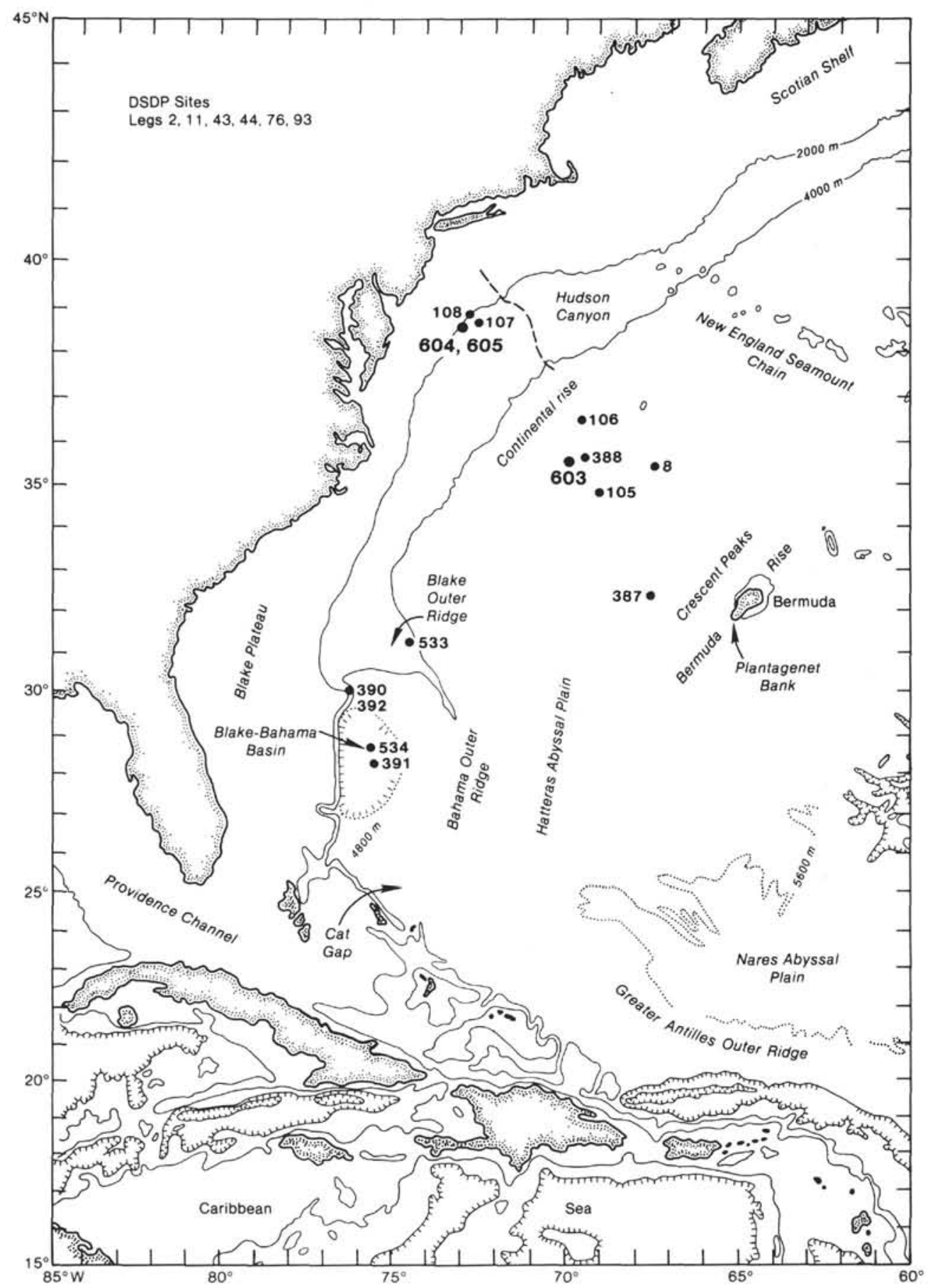

Figure 1. Sites drilled on Deep Sea Drilling Project Leg 93 and earlier legs.

mined to be spines of radiolarians ( $\mathrm{S}$. Kling, personal communication, 1985). These were tabulated in this study and, as their elimination could affect the relative abundance of the sponge spicules, these counts were not excluded from the tables. It is hoped that depicting this form here will prevent other workers from making the same mistake.

"Sinusoidal-styles" (Plate 2, Fig. 10): fragmentary monaxons with a sinusoidal appearance were rare at Site
605. A similar spicule was illustrated by Bukry (1978, pl. 14).

\section{Subclass CERACTINOMORPHA Levi}

\section{Sigmas (strongly curved, or "C"-shaped spicules)}

A wide variety of sigmas were found, particularly at Site 605. Most were "C" or hook-shaped, with the curvature in a single plane, though sigmas curving into the 
Table 1. Relative abundance of siliceous sponge spicules in samples of Eocene sediment from DSDP Site $605\left(38^{\circ} 44.53^{\prime} \mathrm{N}, 72^{\circ} 36.55^{\prime} \mathrm{W}\right.$, water depth $2194 \mathrm{~m})$.

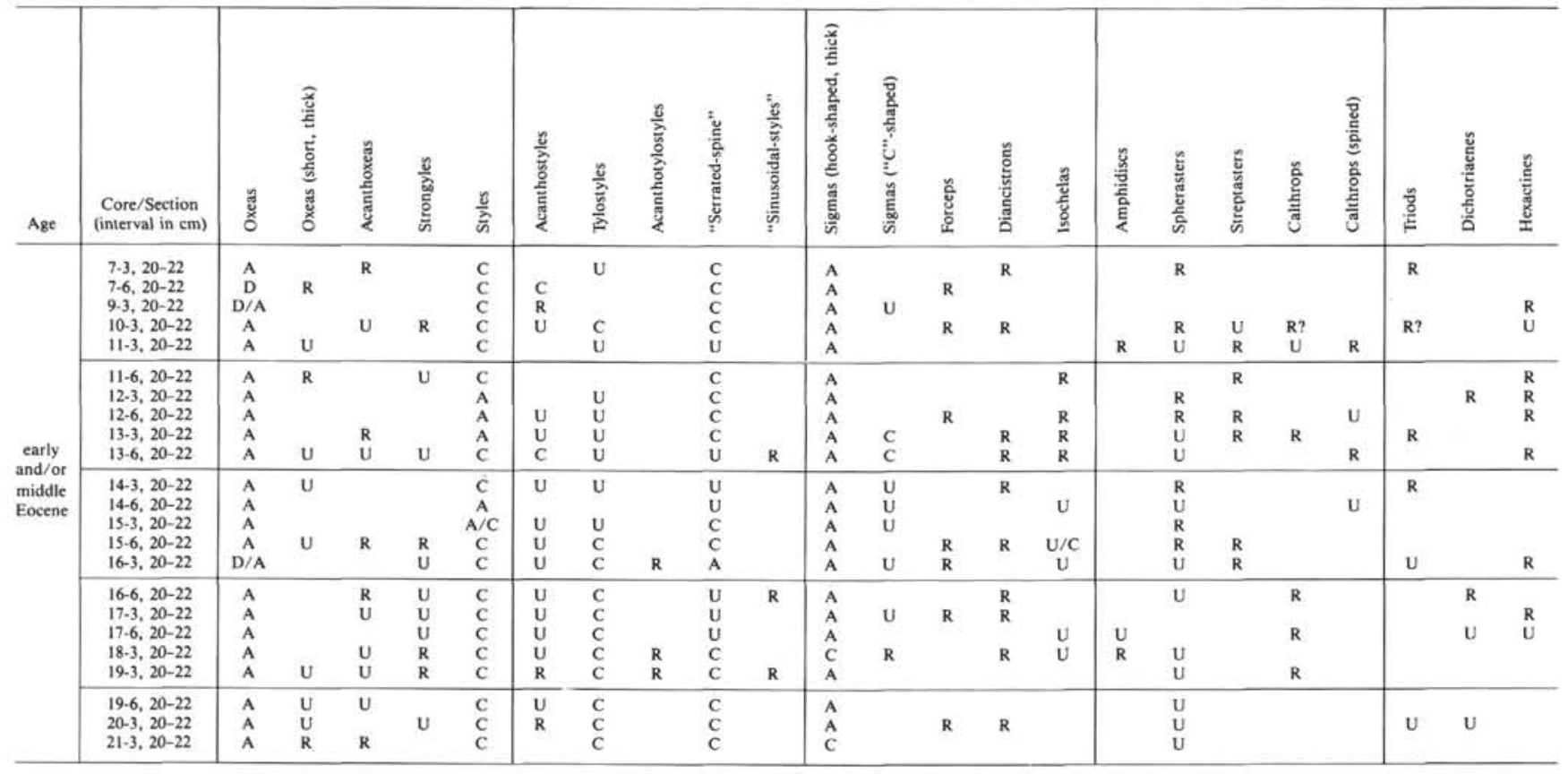

Note: $\mathrm{D}=$ dominant, $\mathrm{A}=$ abundant, $\mathrm{C}=$ common, $\mathrm{U}=$ uncommon, $\mathrm{R}=$ rare.

Table 2. Relative abundance of siliceous sponge spicules in samples of upper Miocene sediment from DSDP Hole 604 $\left(38^{\circ} 42.79^{\prime} \mathrm{N}, 72^{\circ} 32.95^{\prime} \mathrm{W}\right.$, water depth $\left.2364 \mathrm{~m}\right)$.

\begin{tabular}{|c|c|c|c|c|c|c|c|c|c|c|c|c|c|c|}
\hline Age & $\begin{array}{l}\text { Core/Section } \\
\text { (interval in } \mathrm{cm} \text { ) }\end{array}$ & ఊ్ & 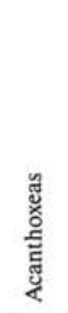 & 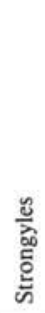 & $\sum_{\tilde{3}}^{2}$ & 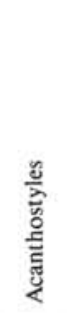 & $\frac{2}{2}$ & 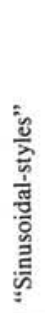 & 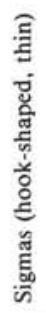 & 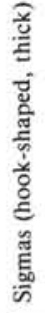 & 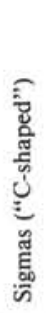 & 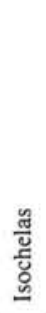 & $\begin{array}{l}\text { 岕 } \\
\text { हैँ } \\
\text { के }\end{array}$ & . \\
\hline \multirow{3}{*}{$\begin{array}{c}\text { late } \\
\text { Miocene }\end{array}$} & $\begin{array}{l}23-1,20-22 \\
23-2,20-22 \\
23-3,20-22 \\
23-4,20-22\end{array}$ & $\begin{array}{l}\mathrm{D} \\
\mathrm{D} \\
\mathrm{D} \\
\mathrm{D}\end{array}$ & $\begin{array}{l}\mathrm{C} \\
\mathrm{C} \\
\mathrm{C} \\
\mathrm{U}\end{array}$ & R & $\begin{array}{c}\mathrm{C} \\
\mathrm{C} / \mathrm{U} \\
\mathrm{U} \\
\mathrm{C}\end{array}$ & $\begin{array}{c}\mathrm{U} \\
\mathrm{U} / \mathrm{U}\end{array}$ & $\begin{array}{l}\mathrm{U} \\
\mathrm{U} \\
\mathrm{U} \\
\mathrm{U}\end{array}$ & & R & $\mathrm{U}$ & $\begin{array}{l}\mathrm{R} \\
\mathrm{R}\end{array}$ & $\begin{array}{l}\mathrm{U} \\
\mathrm{R}\end{array}$ & $\mathbf{R}$ & $\mathbf{R}$ \\
\hline & $\begin{array}{l}25-1,20-22 \\
25-2,20-22 \\
25-3,20-22 \\
25-4,20-22\end{array}$ & $\begin{array}{l}\mathrm{D} \\
\mathrm{D} \\
\mathrm{D} \\
\mathrm{D}\end{array}$ & $\begin{array}{l}\mathrm{R} \\
\mathrm{U} \\
\mathrm{U} \\
\mathrm{C}\end{array}$ & $\begin{array}{l}\mathrm{R} \\
\mathrm{U} \\
\mathrm{R}\end{array}$ & $\begin{array}{c}\mathrm{U} \\
\mathrm{U} \\
\mathrm{C} / \mathrm{U}\end{array}$ & $\mathrm{R}$ & $\begin{array}{l}\mathrm{U} \\
\mathrm{U} \\
\mathrm{U} \\
\mathrm{U}\end{array}$ & R & $\mathrm{R}$ & $\mathrm{R}$ & $\begin{array}{l}R \\
R\end{array}$ & $\begin{array}{l}\mathrm{U} \\
\mathrm{R}\end{array}$ & & $\mathrm{R}$ \\
\hline & $\begin{array}{l}26-1,20-22 \\
26-2,20-22 \\
26-3,20-22 \\
26-4,20-22\end{array}$ & $\begin{array}{l}\mathrm{D} \\
\mathrm{D} \\
\mathrm{D} \\
\mathrm{D}\end{array}$ & $\begin{array}{c}\mathrm{C} \\
\mathrm{C} / \mathrm{U} \\
\mathrm{U} \\
\mathrm{C} ?\end{array}$ & $\begin{array}{l}R \\
R \\
U\end{array}$ & C & $\begin{array}{l}\mathrm{U} \\
\mathrm{U}\end{array}$ & $\begin{array}{l}\mathrm{C} / \mathrm{U} \\
\mathrm{C} \\
\mathrm{C} ? \\
\mathrm{C} ?\end{array}$ & $\mathbf{R}$ & & $\begin{array}{c}\mathrm{R} \\
\mathrm{U} \\
\mathrm{C} ?\end{array}$ & $\mathbf{R}$ & $\mathbf{R}$ & R & $\mathbf{R}$ \\
\hline
\end{tabular}

Note: $\mathrm{D}=$ dominant, $\mathrm{A}=$ abundant, $\mathrm{C}=$ common, $\mathrm{U}=$ uncommon, $\mathrm{R}=$ rare. Question marks indicate uncertainty because of the low abundance of sponge spicules in the sample.

third dimension were found. All strongly curved spicules were included in this category. Many of the oxeas are also curved but to a much smaller degree. There were no intermediates between these two distinct groups.

“ $C$ "-shaped (Plate 5, Figs. 1, 2, 4, 5).

Hook-shaped sigmas, thin (Plate 5, Figs. 3, 8).

Hook-shaped sigmas, thick (Plate 5, Fig. 9): smaller and relatively thicker than the other hook-shaped sigmas, this type was an important background spicule throughout Site 605 .

\section{Order POECILOSCLERIDA Topsent}

Forceps (Plate 5, Figs. 6-7): a spicule with a very deep "U"-shape, found sporadically in Site 605 . See Sollas (1906, p. 222, fig. 109p) for a similar form. 
Diancistrons (resembling a stout "C"-shaped sigma but with the inner margin narrowing to a knife edge and notched; Plate 3, Figs. 4, 5, 7).

Isochelas (curved bar with terminal elaborations; Plate 3, Fig. 8; Plate 6, Figs. 7-11): figures show similar isochelas from different angles.

\section{Subclass TETRACTINOMORPHA Levi}

\section{POLYAXONS (many equal-sized rays radiating from a single point)}

Spherasters (ball-like spicules with pointed spines; Plate 4, Figs. 1, 2): Oxyasters (Plate 4, Figs. 8, 9) were also tabulated in this group.

Streptasters (having spines that radiate from various points along an axis that may be straight or twisted; Plate 4, Figs. 5-6). Some forms which Bukry (1978, pl. 12) considered spirasters were counted within this group. Discorhabds (Plate 4, Figs. 7, 10) were also tabulated in this group.

Calthrops (four tetrahedral rays of equal size; Plate 4, Figs. 3, 4): forms with spines and nonspined rays were tabulated separately.

Triods (three rays of equal size; Plate 4, Fig. 12).

Dichotriaenes (triod with forked rays).

\section{Class HEXACTINELLIDA Schmidt}

Hexactines (six-rays; Plate 6, Figs. 4-5): hexactines were rare and fragmentary at Site 605 .

\section{Subclass AMPHIDISCOPHORA Schulze}

Amphidiscs (rod with recurved, sometimes petaled, disc on each end; Plate 3 , Figs. 1-3, 6, 8?): a variety of amphidiscs having petaled, spear, or anchor-shaped ends were found at Site 605 . Because of their rarity (most of the specimens seen are illustrated on Plate 3 ), these different shapes are tabulated here as a single group.

\section{ACKNOWLEDGMENTS}

I greatfully acknowledge the comments of Dr. Robert M. Finks, Dr. Sherwood W. Wise, Dr. Stanley A. Kling, Mr. Louis Tesar, and Dr. Hsin-Yi Ling. Laboratory support was provided by NSF Grant DPP8414268 . I would like to thank the Deep Sea Drilling Project for providing samples from the Leg 93 material and from other legs for comparative study.

\section{REFERENCES}

Bukry, D., 1978. Cenozoic coccolith, silicoflagellate, and diatom stratigraphy, Deep Sea Drilling Project, Leg 44. In Benson, W. E., Sheridan, R. E., et al., Init. Repts. DSDP, 44: Washington (U.S. Govt. Printing Office), 807-864.

1979. Coccolith and silicoflagellate stratigraphy, northern Mid-Atlantic Ridge and Reykjanes Ridge, Deep Sea Drilling Project Leg 49. In Luyendyk, B. P., Cann, J. R., et al., Init. Repts. $D S D P, 49$ : Washington (U.S. Govt. Printing Office), 551-582. 1980a. Eocene diatoms and siliceous spicules from the northwestern Atlantic Ocean, Deep Sea Drilling Project Sites 417 and 418. In Donnelly, T., Francheteau, J., Bryan, W., Robinson, P., Flower, M., Salisbury, M., et al., Init. Repts. DSDP, 51, 52, 53, Pt. 2: Washington (U.S. Govt. Printing Office), 851-855.

, 1980b. Silicoflagellate biostratigraphy and paleoecology in the eastern equatorial Pacific, Deep Sea Drilling Project Leg 54. In Rosendahl, B. R., Hekinian, R., et al., Init. Repts. DSDP, 54: Washington (U.S. Govt. Printing Office), 545-573.

Dumitricǎ, P., 1973. Cretaceous and Quaternary radiolaria in deep sea sediments from the northwest [sic] Atlantic Ocean and Mediterranean Sea. In Ryan, W. B. F., Hsü, K. J., et al., Init. Repts. DSDP, 13: Washington (U.S. Govt. Printing Office), 829-901.

Ehrenberg, C. G., 1854. Mikrogeologie: Leipzig (Leopold Voss).

Hyman, L. H., 1940. The Invertebrates: Protozoa through Ctenophora: New York (McGraw-Hill Book Co. Inc.), p. 726.

Ivanik, M. M., 1983. Paleogene and Neogene sponge spicules from Sites 511, 512, and 513 in the South Atlantic. In Ludwig, W. J., Krasheninnikov, V. A., et al., Init. Repts. DSDP, 71: Washington (U.S. Govt. Printing Office), 933-950.

Sollas, I. B. J., 1906. Porifera (Sponges). In Harmer, S. F., and Shipley, A. E. (Eds.), The Cambridge Natural History (Vol. 1): New York (Hafner Publishing Co.), 162-242. (1959 reprinting)

Date of Initial Receipt: 13 February 1985

Date of Acceptance 30 July 1985 

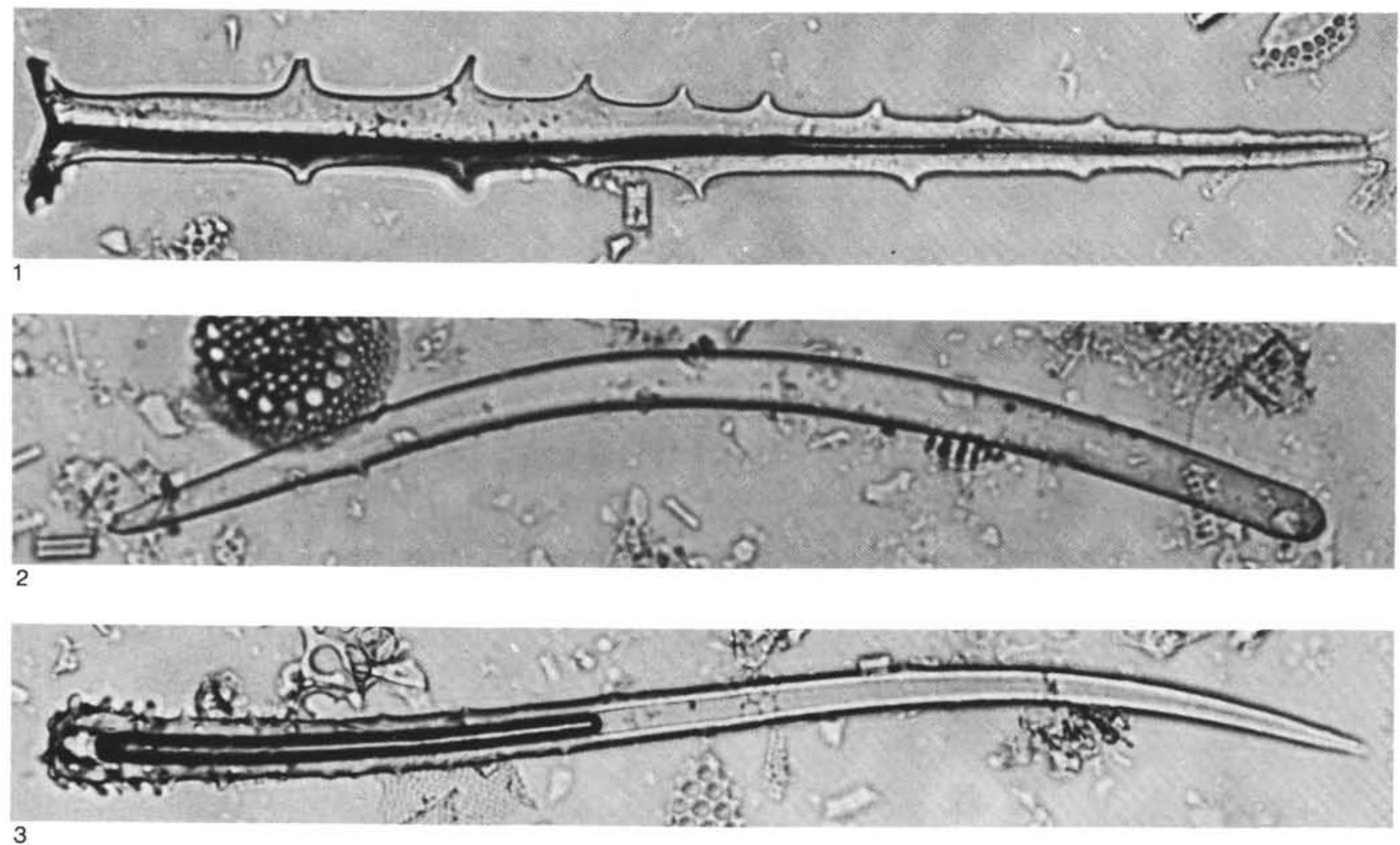

3
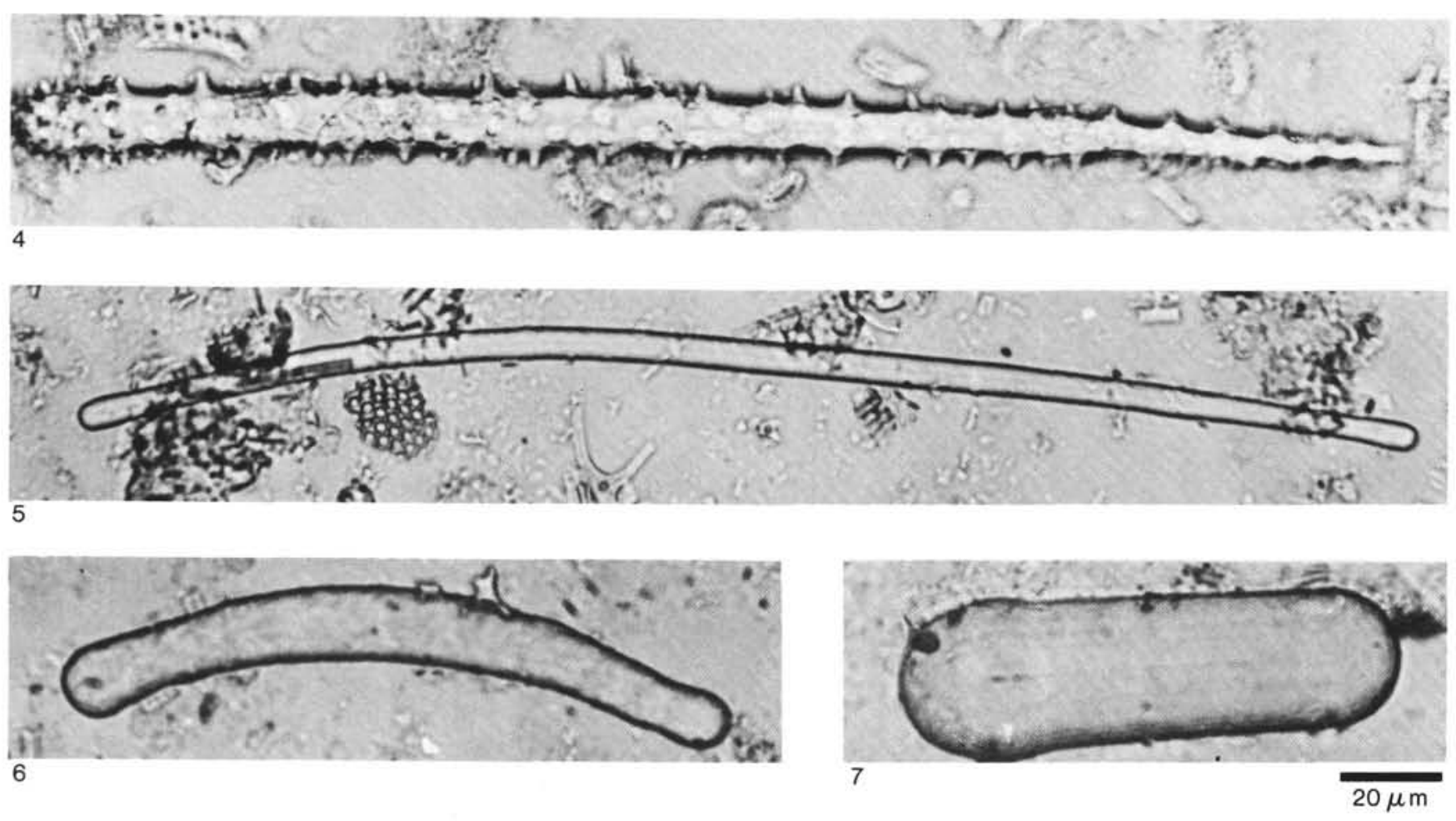

Plate 1. Monaxon sponge spicules from Holes 604 and 605. (Magnification 640×.) 1. "Serrated-spine," Sample 605-12-6, 20-22 cm. 2. Style, Sample 605-16-3, 20-22 cm. 3. Acanthostyle (spinose at rounded end), Sample 605-10-3, 20-22 cm. 4. Acanthostyle, Sample 605-18-3, 20-22 cm. 5-7. Strongyles, (5) Sample 605-16-3, 20-22 cm; (6) Sample 604-25-3, 20-22 cm; (7) Sample 604-25-3, 20-22 cm. 


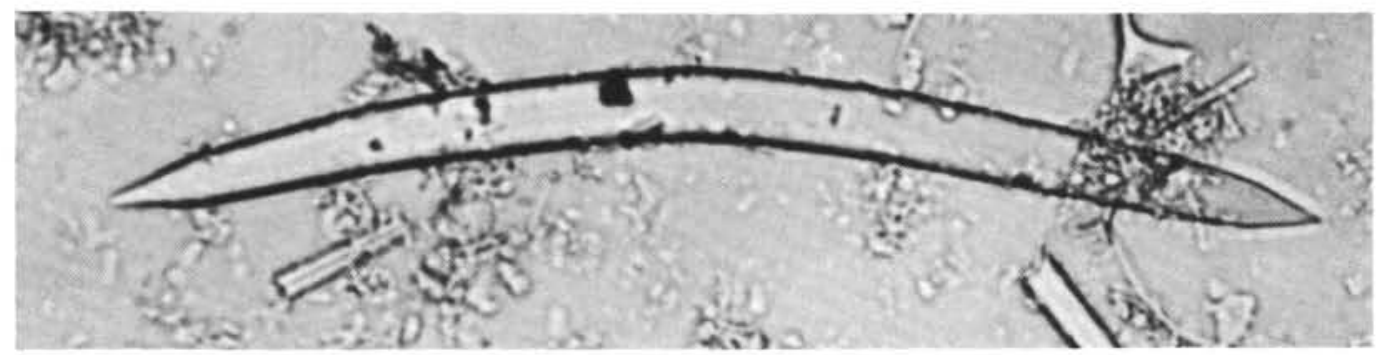

1

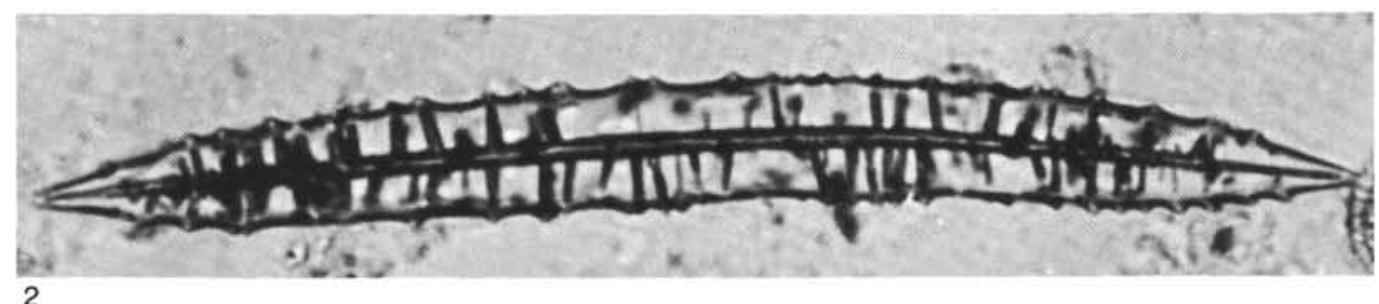

2

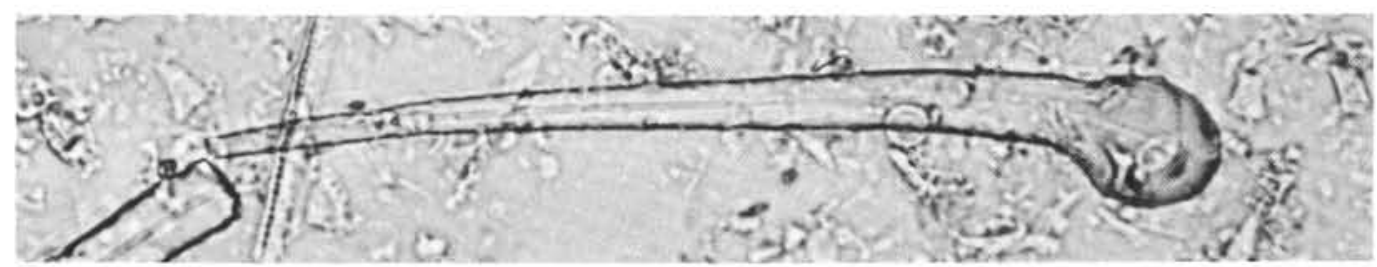

3

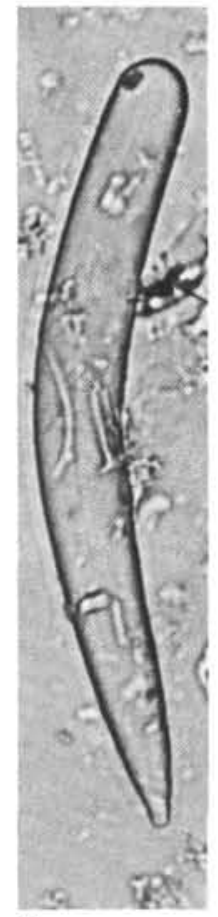

5

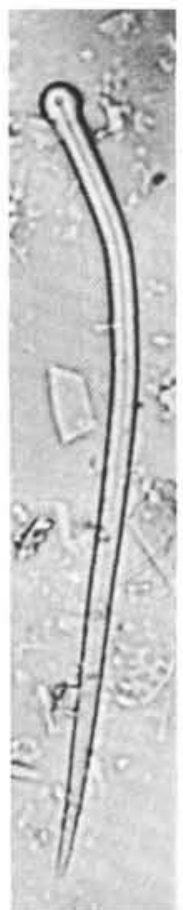

6

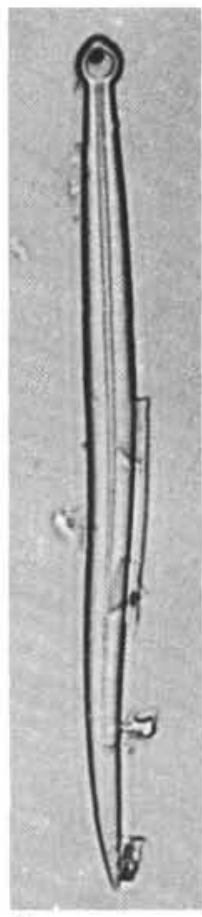

7
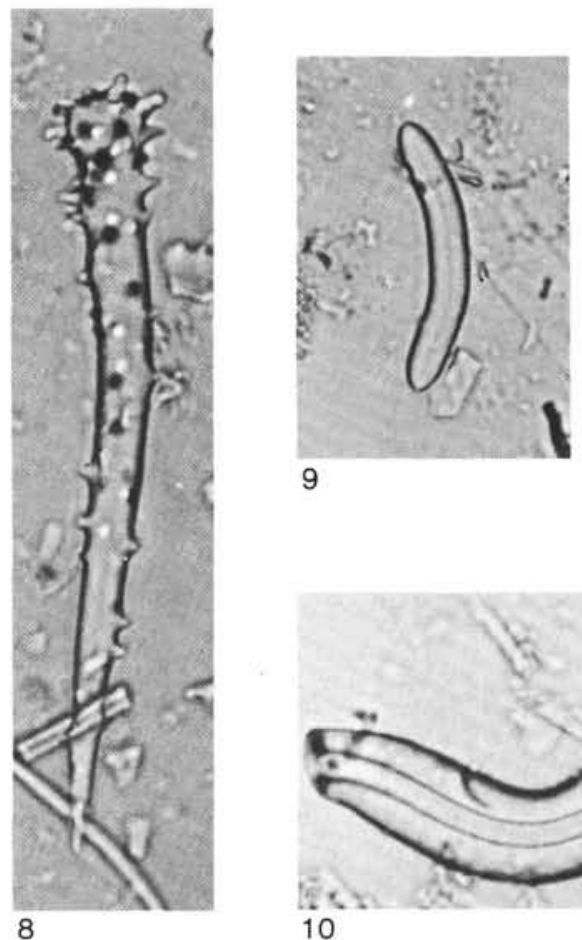

9
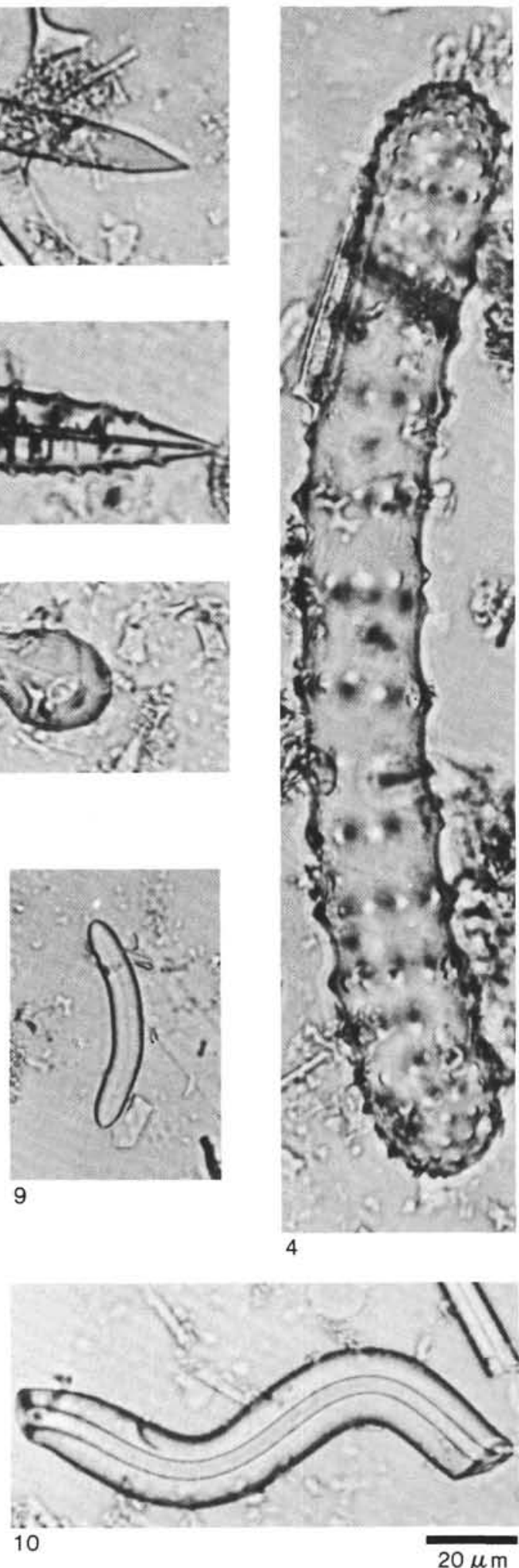

Plate 2. Monaxon sponge spicules from Holes 604 and 605. (Magnification $640 \times$.) 1. Oxea, Sample 605-16-3, 20-22 cm. 2. Acanthoxea (spines not pronounced), Sample 604-25-2, 20-22 cm. 3. Tylostyle, Sample 605-16-3, 20-22 cm. 4. Acanthostrongyle, Sample 605-16-3, 20-22 cm. 5. Style, Sample 605-19-3, 20-22 cm. 6, 7. Tylostyles, (6) Sample 605-20-3, 20-22 cm; (7) Sample 605-7-3, 20-22 cm. 8. Acanthotylostyle, Sample 605-15-6, 20-22 cm. 9. Oxea (short, thick), Sample 605-17-3, 20-22 cm. 10. "Sinusoidal-style," Sample 605-19-3, 20-22 cm. 


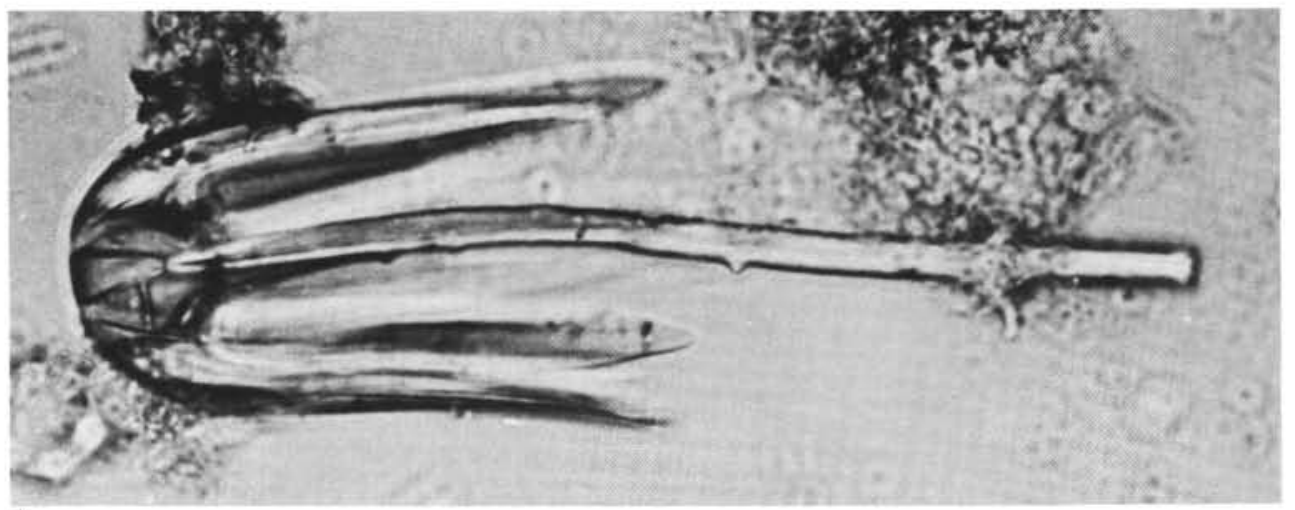

1

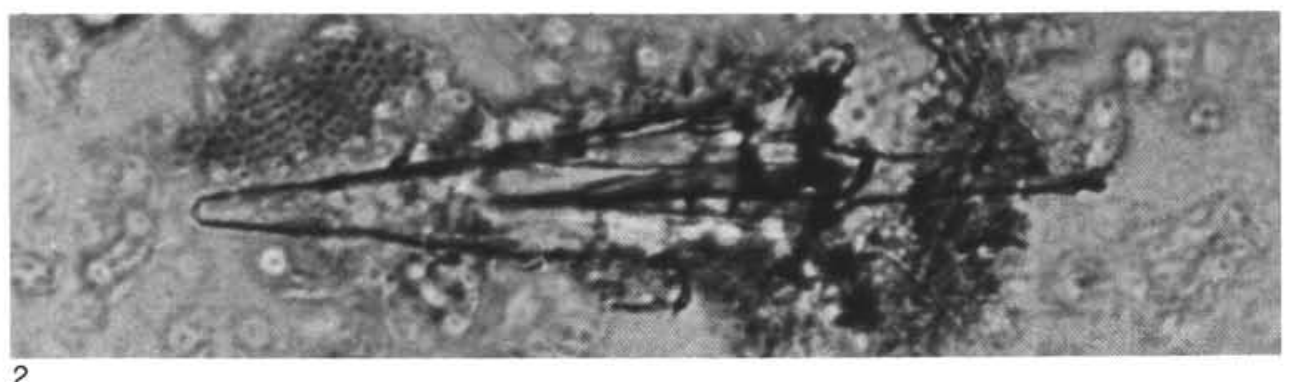
2

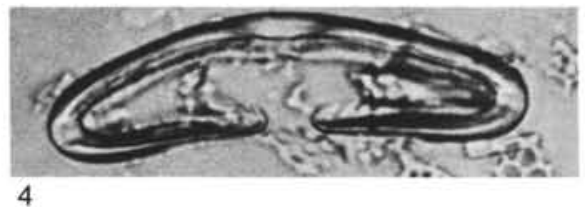

4

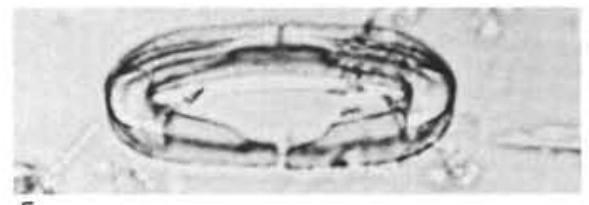

5

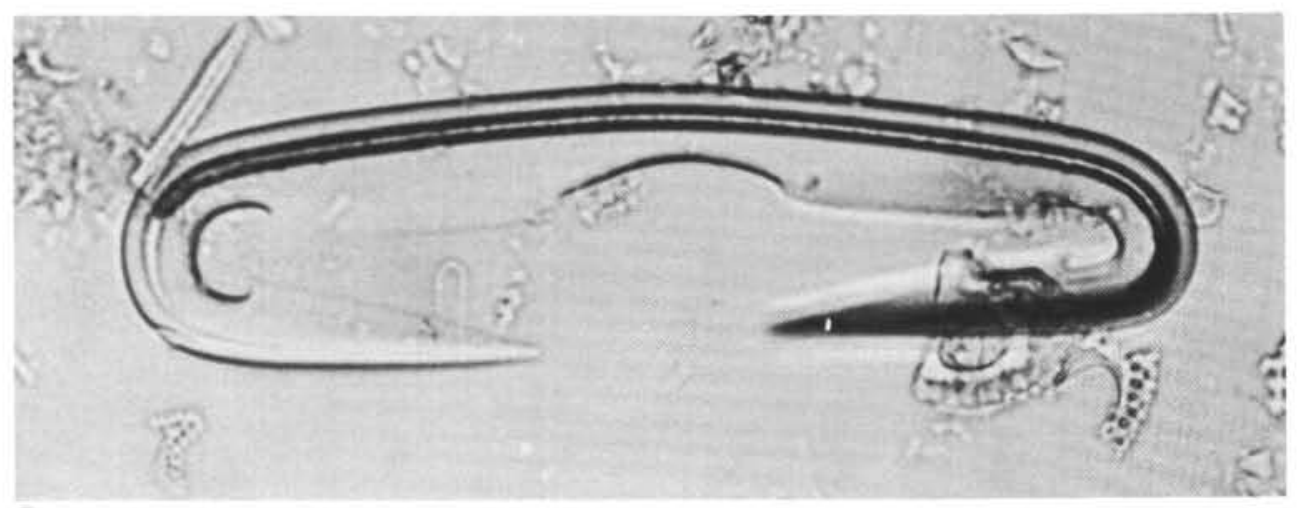

7

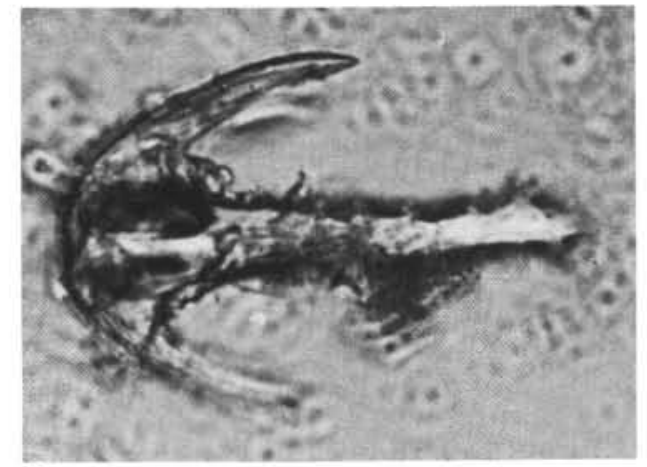

6

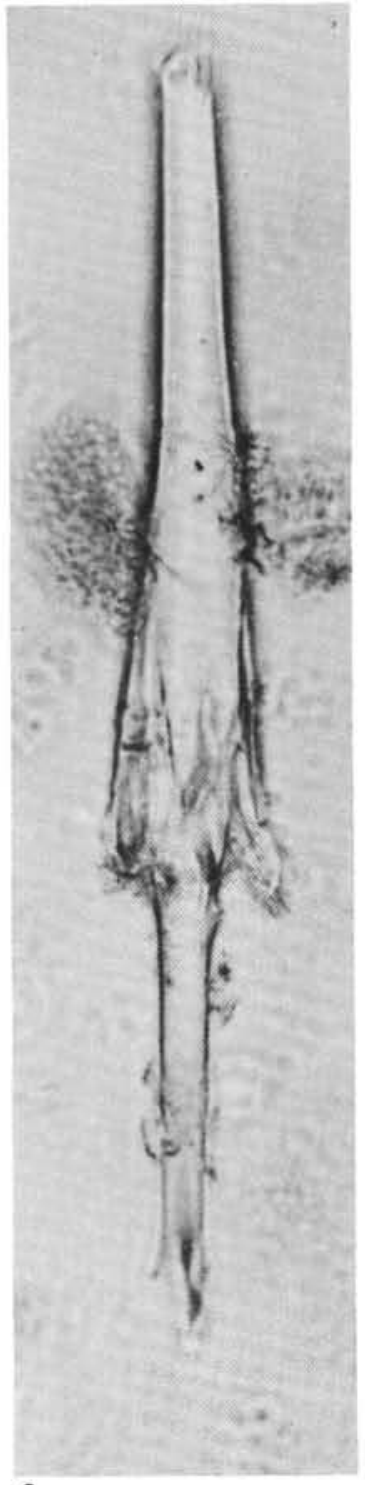

3

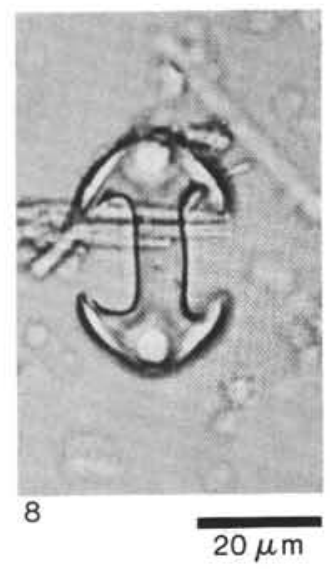

Plate 3. Amphidisc and diancistron sponge spicules from Hole 605. (Magnification $640 \times$.) 1-3. Amphidiscs, (1) with petaled termination, Sample 605-16-3, 20-22 cm; (2) spear-shaped termination, Sample 605-16-6, 20-22 cm; (3) spear-shaped termination, Sample 605-18-3, 20-22 cm. 4, 5. Diancistrons, (4) Sample 605-11-3, 20-22 cm; (5) Sample 605-18-3, 20-22 cm. 6. Amphidisc with anchor-shaped terminations, Sample 605-16-6, 20-22 cm. 7. Diancistron, Sample 605-18-3, 20-22 cm. 8. Isochela (Amphidisc?), Sample 605-11-3, 20-22 cm. 


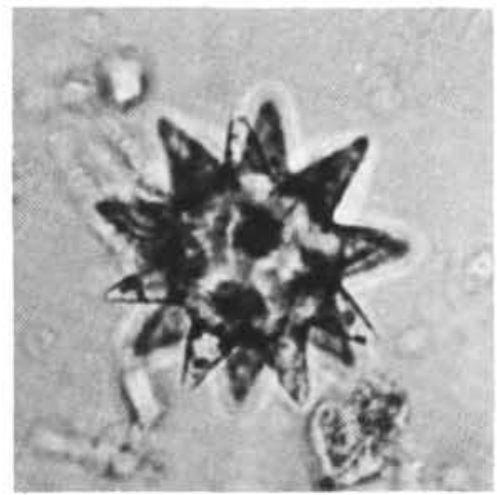

1

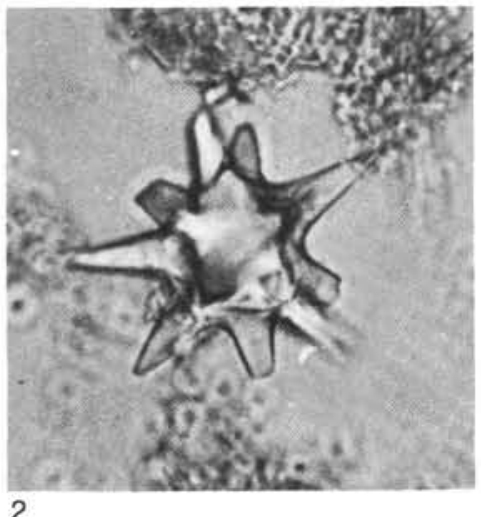

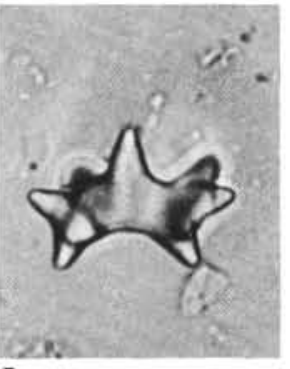

5

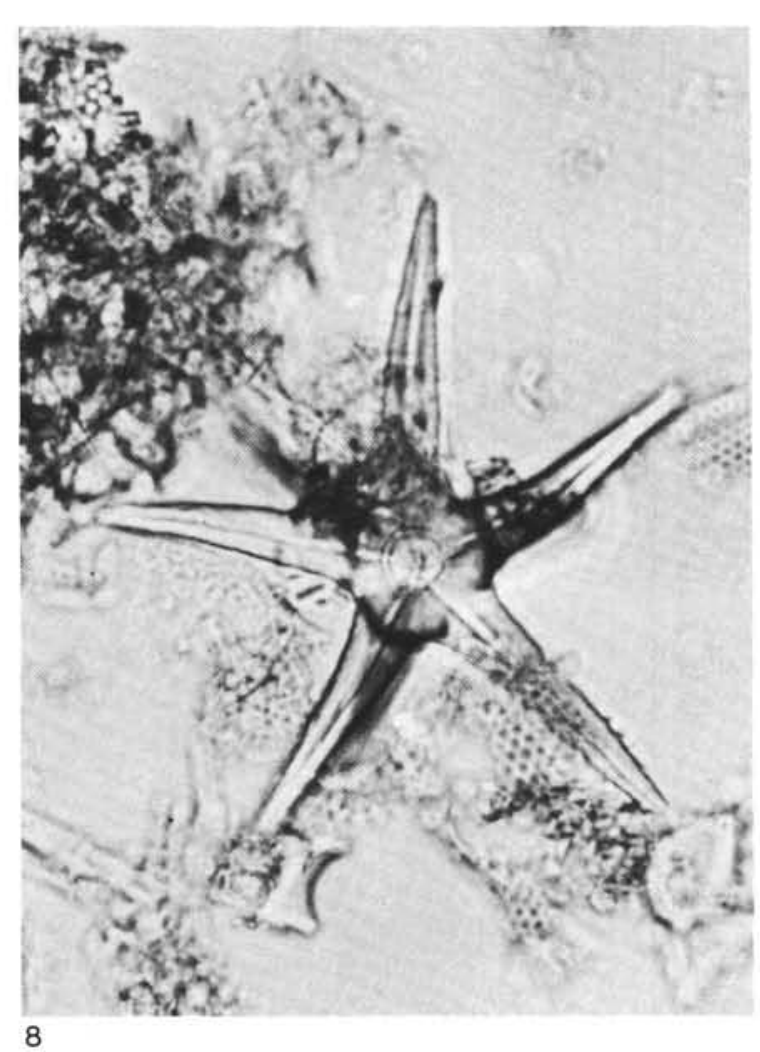

8

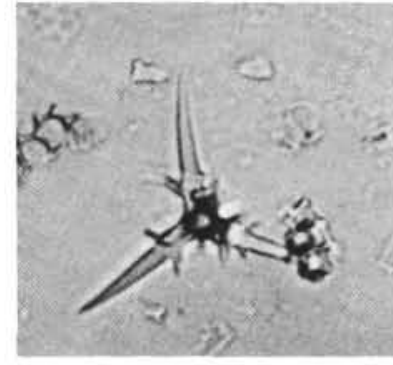

4

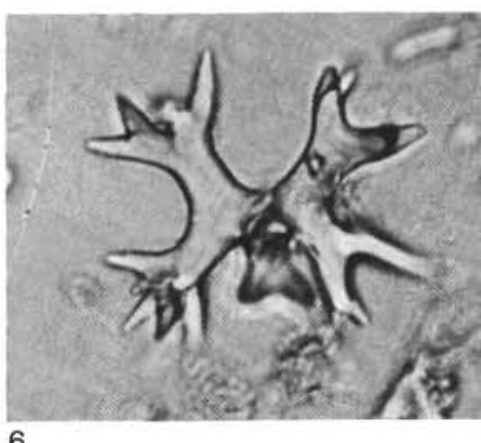

6
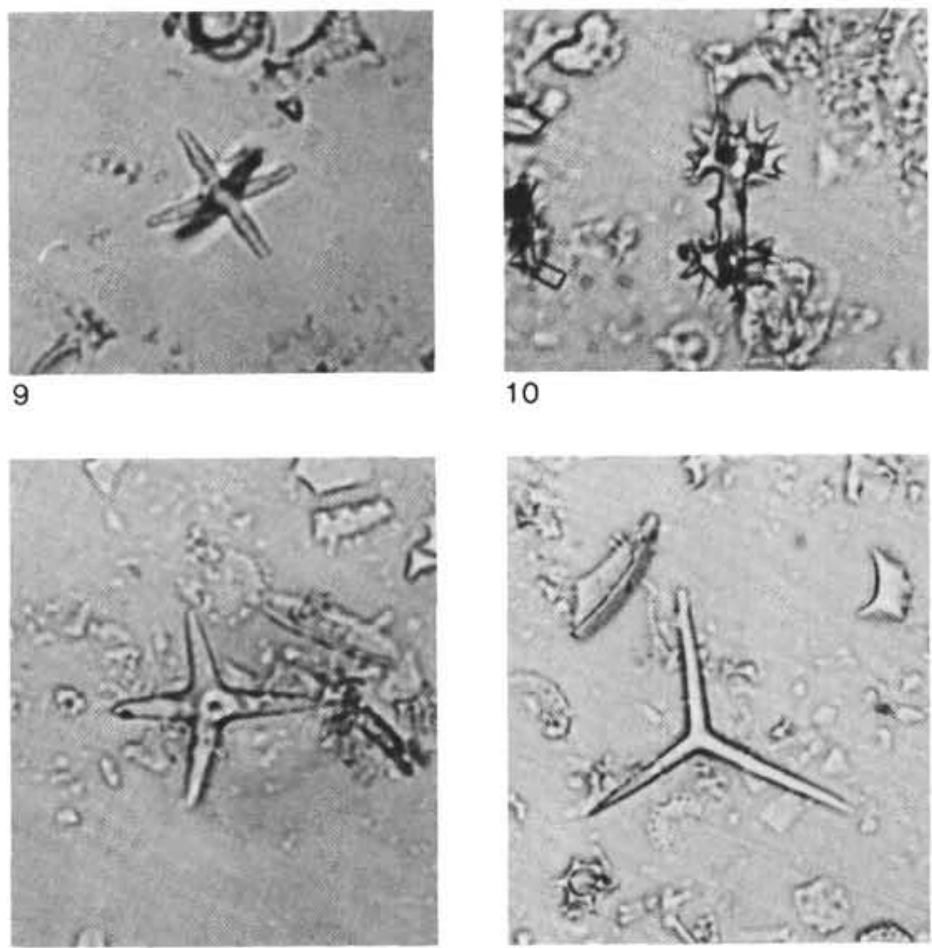

11

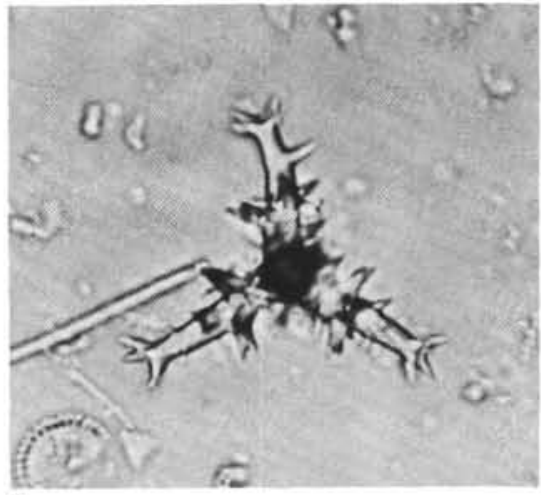

3

Plate 4. Polyaxon sponge spicules from Hole 605. (Magnification 640X.) 1, 2. Spherasters, (1) Sample 605-13-3, 20-22 cm; (2) Sample 605-15-6, 20$22 \mathrm{~cm}$. 3, 4. Caltrops (spinose), (3) Sample 605-13-6, 20-22 cm; (4) Sample 605-12-6, 20-22 cm. 5, 6. Streptasters, (5) Sample 605-12-6, 20-22 $\mathrm{cm}$; (6) Sample 605-10-3, 20-22 cm. 7. Discorhabd, Sample 605-10-3, 20-22 cm. 8, 9. Oxyasters, (8) Sample 605-19-6, 20-22 cm; (9) Sample 605-19-6, 20-22 cm. 10. Discorhabd, Sample 605-16-3, 20-22 cm. 11. Pentactine (?), Sample 605-16-3, 20-22 cm. 12. Triod, Sample 605$19-3,20-22 \mathrm{~cm}$. 


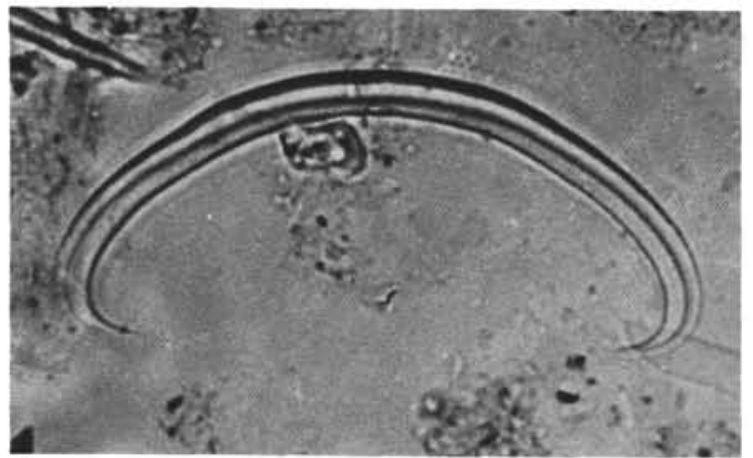

1

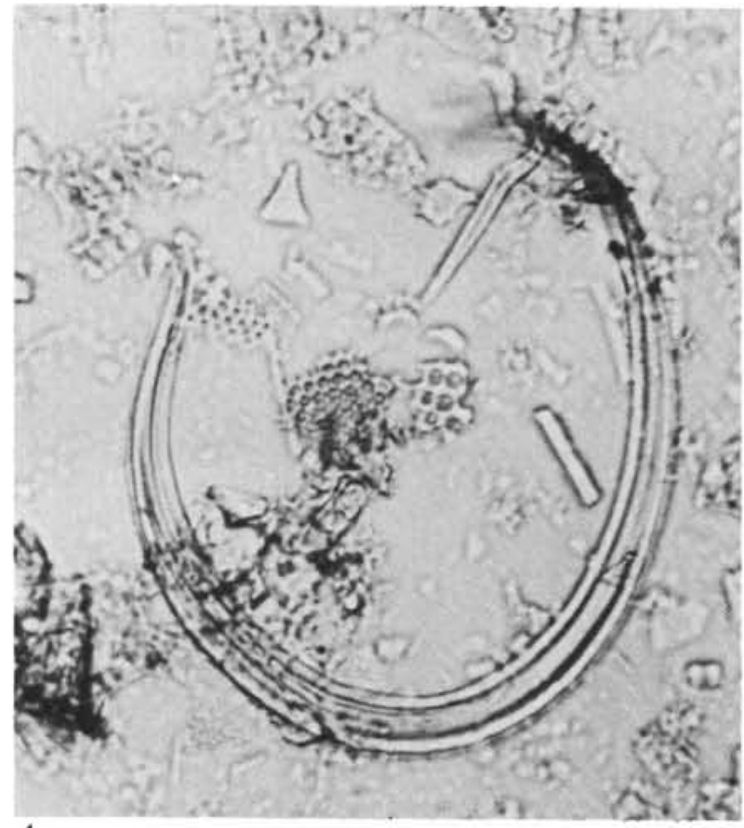

4

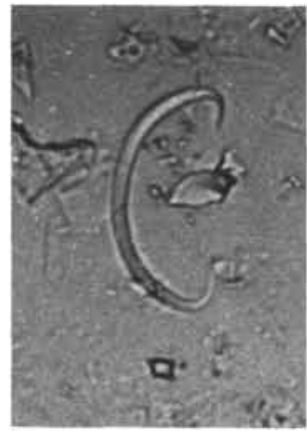

5

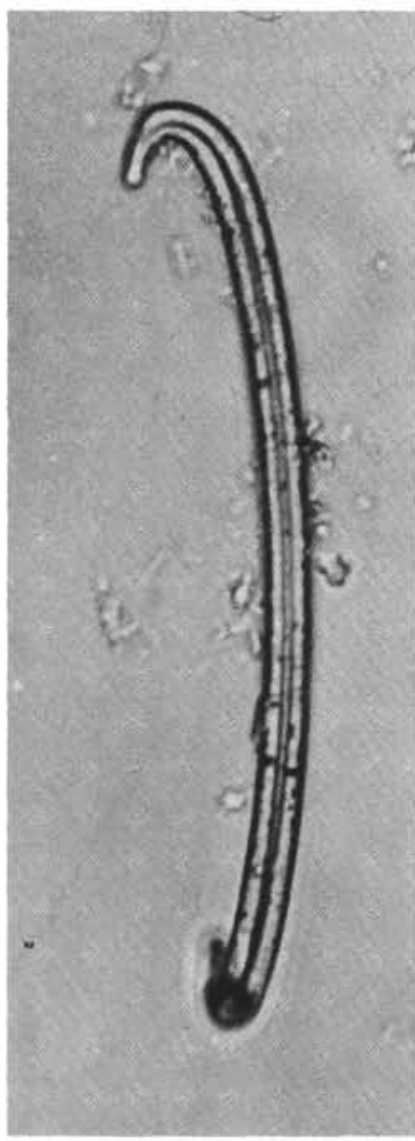

2

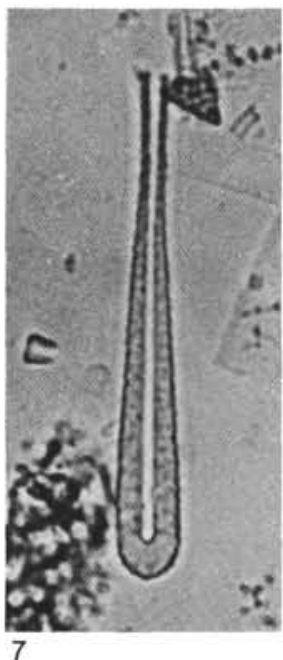

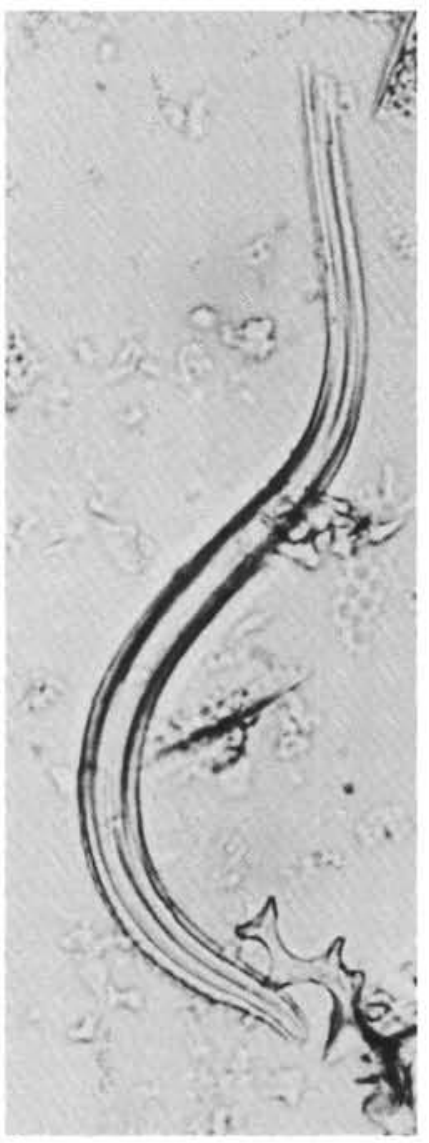
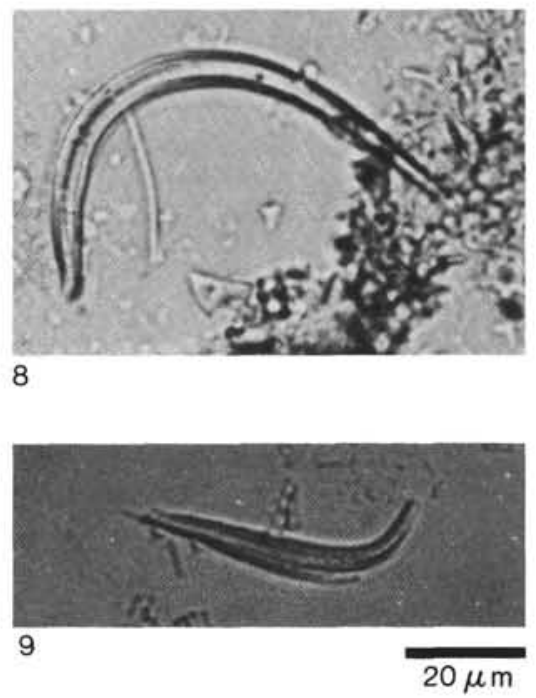

Plate 5. Sigma and forceps sponge spicules from Holes 604 and 605. (Magnification $640 \times$.) 1-5. Sigmas, (1) "C"-shaped, Sample 604-23-2, 20$22 \mathrm{~cm}$; (2) "C"'-shaped, with one limb out of plane of focus, Sample 605-15-3, 20-22 cm; (3) hook-shaped, thin, Sample 605-18-3, 20-22 cm. (4) "C"-shaped, with one end bending out of plane of focus, Sample 605-21-3, 20-22 cm; (5) "C"-shaped, Sample 605-13-6, 20-22 cm. 6, 7. Forceps, (6) Sample 605-17-6, 20-22 cm; (7) Sample 605-17-3, 20-22 cm. 8. Sigmas (hook-shaped), (8) thin, Sample 605-17-6, 20-22 cm; (9) thick, Sample 605-17-3, 20-22 cm. 


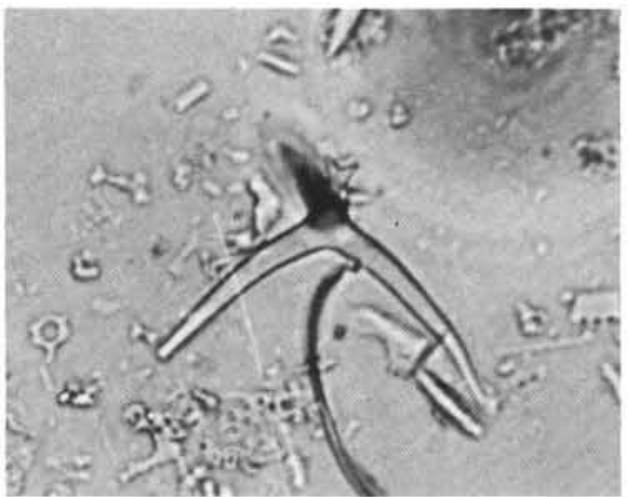

1

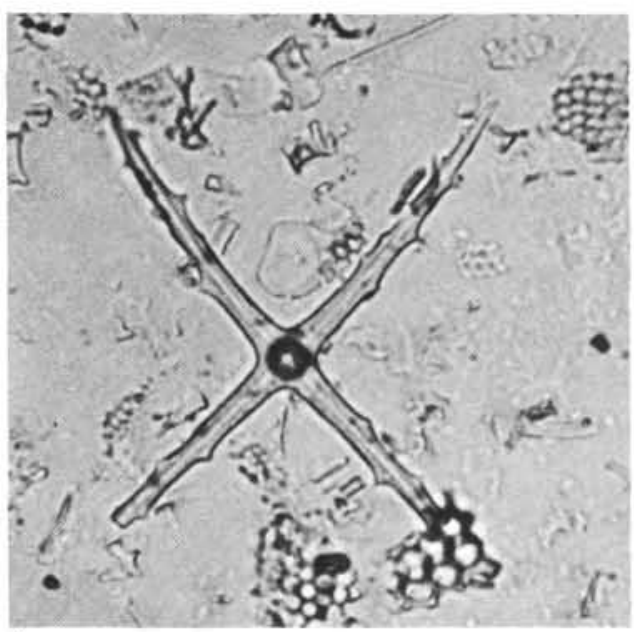

4

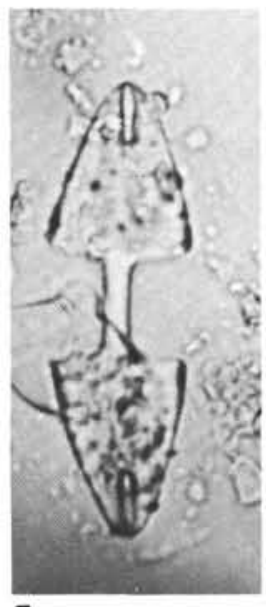

7

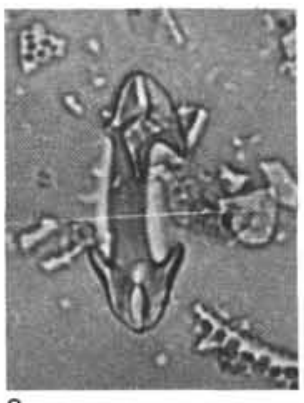

8
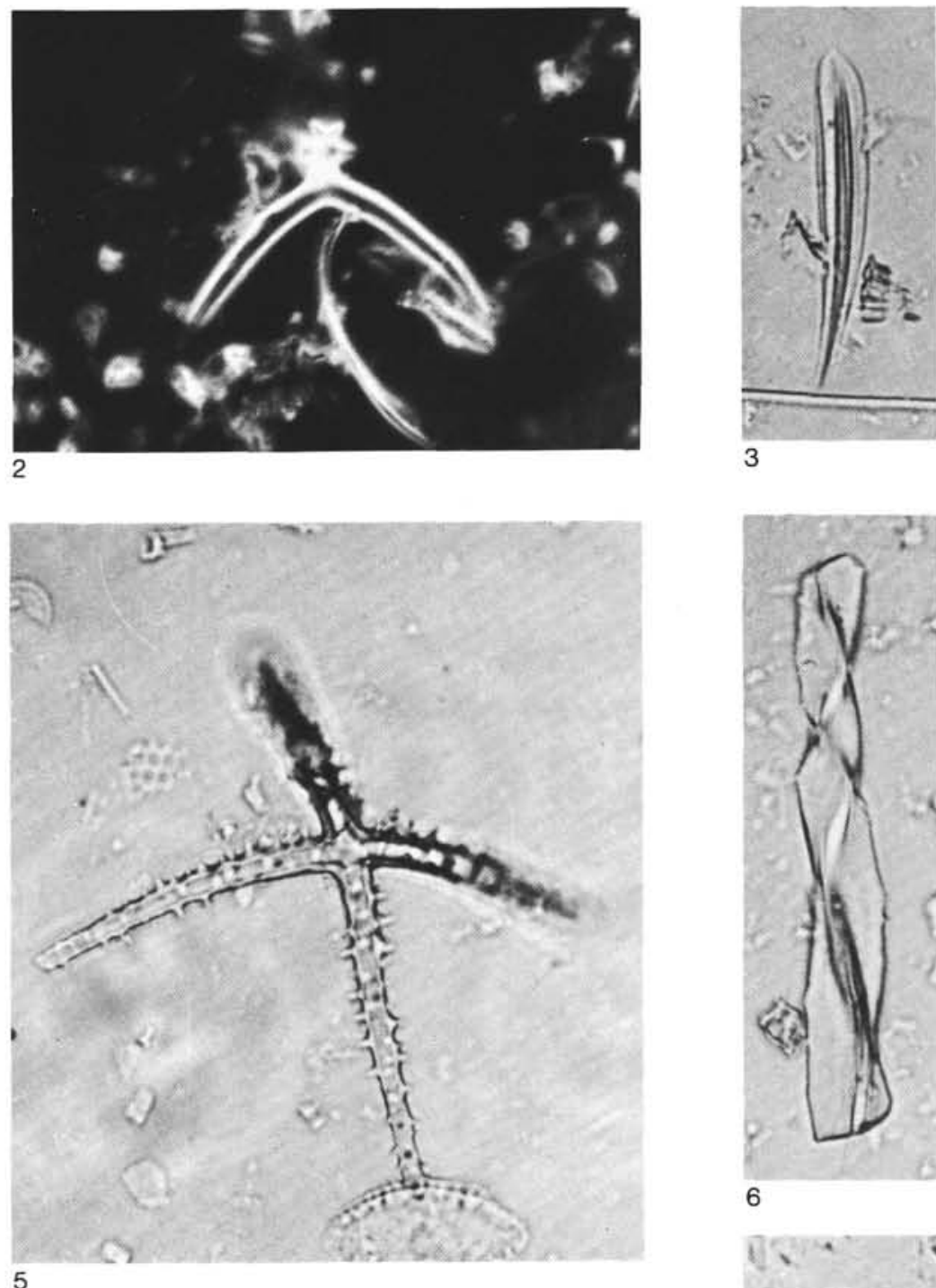

5

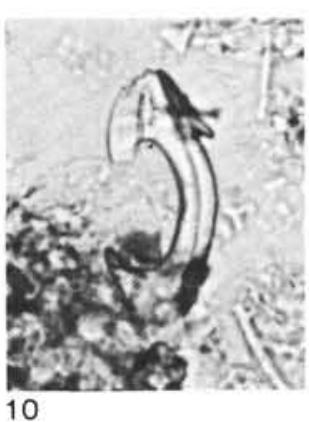

3
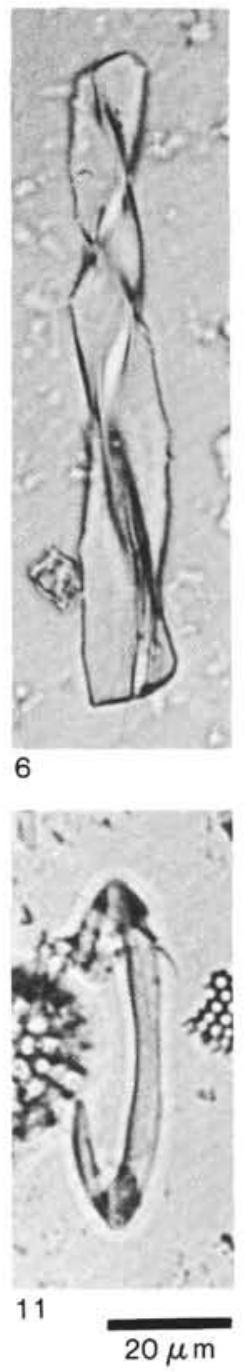

Plate 6. Sponge spicules from Hole 605. (Magnification $640 \times$.) 1, 2. Unidentified sponge spicule, Sample 605-13-3, 20-22 cm; Fig. 2 uses reflected light with dark background. 3. Oxea (asymmetrical), Sample 605-19-3, 20-22 cm. 4, 5. Hexactines, (4) Sample 605-10-3, 20-22 cm; (5) Sample 605-11-6, 20-22 cm. 6. Spirally twisted sponge spicule (?), Sample 605-20-3, 20-22 cm, only example found. 7-11. Isochelas, showing similar specimens from different angles, (7) Sample 605-18-3, 20-22 cm; (8) Sample 605-15-6, 20-22 cm; (9) Sample 605-15-6, 20-22 cm; (10) Sample 605-16-3, 20-22 cm; (11) Sample 605-18-3, 20-22 cm. 